\title{
Research on Current Situation of Network Q \& A Community -An Example of ZHIHU
}

\author{
Juan Wei \\ School of Management, Yulin University, China,719000 \\ 13919363@qq.com
}

Keywords: Network Q\&A community; Current situation; ZHIHU

\begin{abstract}
Nowadays, as the Internet technology becomes increasingly mature and network services are increasingly diversified and personalized, Q \& A websites have emerged because of the needs of the public. The first generation of network Q\&A communities such as " Baidu knows ", "Sina iASK" and so on have greatly enhanced the public access to target audience knowledge and information speed. But in recent years, network problems and shortcomings are gradually emerging in the development of Q\&A communities. In order to encourage the activity of the site users and ensure the quality of the website, there was a real-name registration network Q\&A website, which with the name " ZHIHU " was represented. However, the corresponding problems were followed since 2013. Based on the theory of Q\&A communities, this paper focuses on the current situation of profitability, the status quo of community operation and the status quo of users, and finds out some problems and shortcomings in the development of the network, as well as put forward the corresponding optimization proposal, hoping to provide some references for the follow-up development.
\end{abstract}

\section{Introduction}

In the Internet age, people's cognitive demand for new things is constantly expanding, because each person's work is different, which leads to different perception of the outside world. This means that everyone is familiar with the knowledge of their own work, and there is more or less to the outside world. This contradictory situation makes people eager to learn more about the new things in society, and the network answer platform comes into being and is widely used as the best choice for the answer. According to the 39th report issued by the China Internet information center in January 2017, to December 2016,China's Internet penetration rate has reached 53.2\%, the size of Internet users has reached $731,000,000$, the size of mobile phone users $695,000,000$, with a growth rate of more than $10 \%$ in three consecutive years. January 26, 2011, derived from Quora's knowledge of the net in the domestic line, it initially stepped in the internal phase of the implementation of a strict registration invitation, and providing a knowledge of the net high quality of the question and answer foundation. In March 2013, the net is open to the public, and the number of registered users is rapidly rising from 400,000 to 4,000,000 in less than a year. Network Q\&A community being a special network information exchange platform to the rapid development of the trend into public eye, has plays an important role.

\section{Characteristics of Network Q\&A Community}

Interaction. The Internet itself has a strong interaction, which is characterized by a more specific performance in the network Q\&A community. Because the question-and-answer website itself is a medium of knowledge information dissemination, the information is established between the questioner and the respondents, and the communication between users and users is everywhere. In addition, thanks to the technical support of web2.0, the information screening mechanism of the network is more perfect, and fully guarantee the authenticity of the website information. ZHIHU has prompted its knowledge to spread and the communication channel of information. 
Sharing. Network Q\&A community is fundamentally based on the content that the user is interested in, and users can pay attention to the issues, topics, and even users, which accelerates the transmission speed of knowledge information in the Internet age. Whenever a user asks a question, the answer not only provides the answer to the question, but also shows the other users who pay attention to this problem. The information is shared between users who are interested in the same topic. American writer ClayShirky said in "cognitive surplus: the power of free time", educated and free-time people, with rich knowledge background. At the same time, there is a strong desire to share, and these people are gathered together to produce a huge social effect. Therefore, in terms of information transmission speed or user demand, ZHIHU has very strong information sharing.

Professional. In the beginning of the network, the establishment of the network is mainly aimed at a number of deep problems to carry out professional answers, in answer not only to pay attention to the answer to the professional and logic of the answer. Moreover, there is a high demand for the question of the questioner, and there is no high-quality and meaningless answer to the question. Because the first batch of users in ZHIHU is generally a professional person with higher knowledge literacy, their answer has fully guaranteed the authenticity and professionalism of the question and answer of ZHIHU. Ensure the high-quality community atmosphere of the website platform.

Social. In the network Q\&A community, the life background and the knowledge skills of the user are fully determined by the number and quality of their questions, and also show their personal charm at the same time. On the platform of ZHIHU, the user, in the process of browsing, is automatically classified according to the quality of the answer, which determines whether the social relationship extends from the line to the line. Some of the users who have similar backgrounds and interests have a similar view of the problem. This part of user is very easy to get through such a link to the line of social relations gradually extended to the real life, and to keep this kind of friendly relationship development.

\section{Current Situation of Development of ZHIHU}

With the growing maturity of Internet technology, many new ideas and new models have been put forward in the network. We have always known that the business concept of the high-quality Q\&A platform has achieved its good industry reputation, especially since ZHIHU's opening up in 2013, the user group has been expanding day by day. In the Chinese socialization network Q\&A industry has the significant influence.

Profit model. The net official said that the future of its profit model will not be too different from traditional enterprises, but also advertising profit-based. ZHIHU is mainly dependent on advertising and content operation to obtain profit, after several years of operation, the content operating system of ZHIHU is gradually improved, online products are constantly updated. The operating system is increasingly mature, which brings great influence to ZHIHU, and also brings more profit. In view of the needs of network to high quality answer to the community, ZHIHU's operating team has been a very restrained attitude towards the station. This is mainly reflected in the number of advertising on the site and the quality of the strict control. The ZHIHU has always been a very strict screening program, users in the first page of the website will often have only 1 to 2 patch ads, and the main color is blue. For the high-quality users who know the net, it is one of the important reasons why they remain active so far. Although this approach has guaranteed the user's good use of feelings, but only to know that the net brings very little profit. On the other hand, we know that the net efforts to construct the content ecosystem, namely, the high-quality original content of the station to carry on the operation of the two processing, the ability to spread the value and profitability, so that the knowledge of the net profit from the net. Such as in 2016, it provided institutional account, the knowledge of the bookstore, and so on, but this income is mostly given to the content of the original author, left to know that there are fewer parts of the net. There is no ability to maintain the operating expenses of the website, as far as it is concerned, the profitability of the net is relatively weak.

Community operation. Knowledge network is currently ranked in the industry in general in a stable trend. By January 2017, ZHIHU has already had more than 65,000,000 registered users, averaging 
$1,8,500,000$ active users every day to visit and use. For the whole 2016, Internet users have raised more than 6,000,000 questions, writing more than 23,000,000 answers and 1,510,000 articles. With the increasing number of users since the opening of ZHIHU in 2013, its influence on the Chinese network and Q\&A community has been expanded, and the ranking is increasing in the same industry. However, in Alexa website rankings, the net ranking is continuing to decline, only a month of time from the original 107 to 136, which shows the development of the net has slowed down. Because ZHIHU is currently located in a completely competitive market, sometimes a small change in function, can trigger users to make different behavior choices, thus affecting the whole market. To do this, we know that ZHIHU based on different market user needs to develop different market strategies.

Community users. ZHIHU has been pursued by users as the center of the community operation. In the community promotion, the web page is a simple, brisk color and page display to the user with a rational, interesting impression; In user maintenance, we know that ZHIHU launches the public domain editor plan, report mechanism, modify and delete function 3 user management mechanism. In addition, users will automatically feedback to the background of the interested topic which is selected by the user when they enter ZHIHU, and the background will be sent to use regularly according to the topic of the user's interest by e-mail to enhance the user's memory, thus improving the user's viscosity. According to the user's active degree, the net user can be divided into three types, one is active, and a high-quality user who makes great contributions to the content of the community. This batch of users will generally be the elite of some industry or the professionals in a field, they produce a high-quality, reliable, and often have a lot of followers in the community; one is active, occasionally production and answer content or comment on the middle layer of the user, this part of the content of the user content is not high; The other is active low, almost non-production of ordinary users, this part of the user, although the use of knowledge is more frequent,? But they are more of the high-quality questions that are produced by other users of the community, and the number of questions that the community provides for a variety of reasons is not much.

\section{Problems of ZHIHU's development}

High-end image is weakened. In order to ensure the quality of community users, the quality of the community is also guaranteed. As ZHIHU opening up in 2013, the number of problems in the number of users has become increasingly revealed, such as a variety of meaningless answers will be uninterrupted. Contrary to the original intention of the creation. When a large number of low-level users come in, the anonymous community environment in protecting the privacy of the user also greatly broadened their activities, although the user knows the rules of the game inside the community. But by the lure of self-interest, as long as there is a person who breaks the rules, then the following is a good reason why does not obey the rules of the game. And with the increase of the number of ordinary users, the number of problems users will also increase, this kind of violation of the rules of the game will be more and more likely. It is enough to see the scale development of the net to a certain extent weaken the inherent high-end image of the net.

The copyright protection mechanism is not perfect enough. With the development of network technology and the popularization of network equipment, ZHIHU provides a shortcut for the illegal behavior of some speculators. Such as the early micro-blog marketing number or Wechat public number, without the author's permission to the long-term text or screenshots to be reproduced in a number of high-quality answers. To a certain extent infringe on the interests of the author. The web is a platform for free sharing of knowledge between users, such as knowing that the main station will be on the site of some of the high-quality content to be known as "the weekly." In the form of e-books, put on the kindle platform for free download reading, but this does not mean that the original content of Internet users can be used by others. Reprint to seek hits or business interests. When the copyright of high-quality users has been infringed on many times, its self-identity and social identity will weaken, and the enthusiasm of content creation will fall. There is a decrease in the number of high-quality questions in the community.

The number of high-quality users is too small. Due to the early application of the invitation system, ZHIHU of the early user structure is single, most of ZHIHU, or in other areas of the elite group. Although 
the post-open registration has widened the source of the network community user sources, the community has attracted the elite users of all walks of life, but the increased users are mostly with no attention, no answer, no approval, and at the same time, active high-quality and high-quality answers to high-quality users with a decrease in the proportion of high-quality. The high-quality users of ZHIHU have contributed to the quality of the community $80 \%$, so the relatively small quality users have led to the shortage of high-quality original content of the community. There are certain limitations to the long-term stable development of ZHIHU.

The user maintenance system is not perfect. Since the beginning of 2014, the phenomenon of high-quality user loss, especially with a number of well-known users to leave the entire knowledge of the web site users have decreased significantly. Although ZHIHU has repeatedly emphasized the high level of content, but still can not control the increase of the user base, the source of information from different sources of the community is low. A state of disorder appears as ZHIHU has even been seen by users through private letters to purchase transactions, which makes the understanding of serious and friendly knowledge into an atmosphere suddenly become no longer pure, valuable information becomes rare while garbage information is redundant. Because the rights and interests of high-quality users have not been maintained in time, a large number of high-quality users gradually left the knowledge of the net, and some more directly delete themselves in the knowledge of all the questions and answers on the Internet. The loss of a lot of high-quality content has caused great loss to the development of ZHIHU.

\section{Suggestions of ZHIHU's development}

Create a good community atmosphere. The domestic network Q\&A community are faced with rubbish information, network violence, infringement and so on. As a typical representative of the high-quality Q\&A community in China, how to provide a good community atmosphere for users is the key to the development of the community. ZHIHU can attract more users who are willing to share knowledge, experience and insight. Therefore, ZHIHU can continuously improve its own website system, from multiple channels to the compulsory or semi-compulsory delete or shield some non-practical questions and answers, thus purifying the community environment; Secondly, we know that ZHIHU can effectively promote the user's reward and punishment mechanism, with the user's vital interests to restrict its answer, and to promote the harmonious civilization of the community and answer environment construction; Finally, ZHIHU should continue to uphold its content to create high-quality content to attract the user's high-quality Q\&A community.

Perfect copyright protection system. Although ZHIHU has been declared to protect the copyright of the user, it is also known to the network platform to establish a network of copyright, but did not really solve the copyright problem. Therefore, ZHIHU should do the work of the intermediary, to establish an effective and feasible connection path for the original user and other social media platform of the original content. To ensure the actual interests of users; In addition, it is free to provide legal assistance to the original author for free, and fully guarantee the rights and interests of individual knowledge and creation on other media platforms.

Actively cultivate high-quality users. Most users are common users, but this part of user also has some industry elite or academic experts who are fully capable of answering. But for a variety of reasons, they were not strong enough to answer. For this reason, ZHIHU can be targeted to set up a number of rewards and punishment system, from material rewards or spiritual incentives to stimulate the lower level users to answer the will, and improve their answer to the participation degree. In addition, ZHIHU can add to the problems or topics of ordinary people's life, and the common users have a sense of resonance, so that ordinary users want to talk, to say in order to find more potential high-quality users in ordinary users, expand the scope of the net quality users, increase the number of active users, and achieve the purpose of rich community content.

Further improve the user maintenance system. To ensure that the user's participation degree, ZHIHU also should carefully consider the user's media literacy, appropriate to the user for certain levels of refinement. For different level users to set the corresponding reply, such as browsing level, comments, and other related issues. The settings of permissions, on the micro, can guarantee the quality of the 
answer, maintain the rights and interests of high-quality users, the macro level can be more accurate to grasp and meet the user needs. Because the full equality of equal rights in the Internet information is actually given to those high-quality content makers of the greater inequality. For example, some excellent answers have been sunk to the bottom because of too much opposition to the bottom, and a lot of unanswered questions have been pursued and responded by a lot of people, some even thousands. Therefore, the reasonable establishment of the user's rights to a certain extent maintain the interests of the community high-quality users, and also protect the community fair and harmonious discussion environment.

\section{References}

[1] Lei Yu. Problems and Development Suggestions of Net Existing in User's Perspective, New Media Research, Vol. 3 (2015) No.5, p.3-4.

[2] Li Dan. Comparative Study of Sino-American Network Q\&A community: Examples of Quora and ZHIHU, Youth Journalist, Vol. 7 (2014) No.26, p.19-20.

[3] Liu Mengli. Research on Four Kinds of Scientific Communication Mode of Network Q\&A Platform (MS., Guangxi University, China 2014), p.12.

[4] Hao Zhenjiao. On the Advertising Value and Marketing Strategy of Social Networking Sites (MS.,Hebei University, China 2015), p.30.

[5] Jia jia. Evaluation of Answer Quality of Social Q\&A Platform, Journal of Information Resources Management,Vol. 9 (2012) No.2, p.19. 